
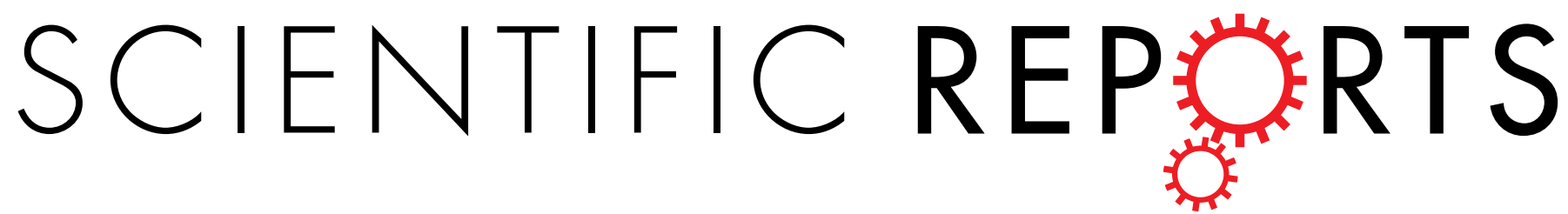

OPEN

Received: 31 March 2016

Accepted: 30 June 2016

Published: 25 July 2016

\section{Two $h A T$ transposon genes were transferred from Brassicaceae to broomrapes and are actively expressed in some recipients}

\author{
Ting Sun ${ }^{1,2,3}$, Susanne S. Renner ${ }^{4}$, Yuxing Xu ${ }^{1,3}$, Yan $\operatorname{Qin}^{1,5}$, Jianqiang Wu ${ }^{1}$ \& Guiling Sun ${ }^{1,2,5}$
}

A growing body of evidence is pointing to an important role of horizontal gene transfer (HGT) in the evolution of higher plants. However, reports of HGTs of transposable elements (TEs) in plants are still scarce, and only one case is known of a class II transposon horizontally transferred between grasses. To investigate possible TE transfers in dicots, we performed transcriptome screening in the obligate root parasite Phelipanche aegyptiaca (Orobanchaceae), data-mining in the draft genome assemblies of four other Orobanchaceae, gene cloning, gene annotation in species with genomic information, and a molecular phylogenetic analysis. We discovered that the broomrape genera Phelipanche and Orobanche acquired two related nuclear genes (christened $B O$ transposase genes), a new group of the $h A T$ superfamily of class II transposons, from Asian Sisymbrieae or a closely related tribe of Brassicaceae, by HGT. The collinearity of the flanking genes, lack of a classic border structure, and low expression levels suggest that $B O$ transposase genes cannot transpose in Brassicaceae, whereas they are highly expressed in $P$. aegyptiaca.

Horizontal gene transfer (HGT) refers to the transfer of genetic material to non-offspring genomes ${ }^{1-3}$. In prokaryotes, it is a vital source of evolutionary novelty. In eukaryotes, the role of HGT in physiological and ecological adaptations has become increasingly clear with examples now known from protists, fungi, animals, and plants ${ }^{3-12}$. For instance, a neochrome originating in hornworts was transferred horizontally to ferns, where it may have played a role in the diversification of modern ferns ${ }^{5}$. Most HGTs in plants involve mitochondrial DNA transferred to nuclear or mitochondrial genomes, while HGTs of nuclear and plastid DNA are rare, with the nuclear transfers mostly involving parasitic plants ${ }^{6-12}$. Thus, the root parasite Striga hermonthica (Orobanchaceae) has acquired an unknown protein-coding gene from its host Sorghum (Poaceae), apparently via an RNA intermediate ${ }^{10}$; the root parasite Rafflesia cantleyi (Rafflesiaceae) has acquired several dozen actively transcribed protein-coding genes from its obligate host Tetrastigma rafflesiae $(\text { Vitaceae })^{6}$; the root parasites Orobanche spp. and Phelipanche spp. (Orobanchaceae) and the shoot parasite Cuscuta pentagona (Convolvulaceae) have acquired albumin genes from legume hosts ${ }^{11}$, and Phelipanche aegyptiaca (Pers.) Pomel (formerly Orobanche aegyptiaca Pers.) and Cuscuta australis further acquired strictosidine synthase-like (SSL) genes from Brassicaceae hosts ${ }^{12}$. The frequency of HGT in parasitic plants is attributed to parasites establishing intimate connections with their hosts through haustoria that enable them to take up water, nutrients, and macromolecules including mRNAs ${ }^{13}$ and sometimes also DNA.

Transposable elements (TEs) constitute the main components of most eukaryotic genomes, occupying 3-20\% of fungal, $3-45 \%$ of metazoan, $50 \%$ of human, and $80 \%$ or more of plant genomes ${ }^{14}$. They translocate to new positions in the same genome by either a copy-and-paste mechanism involving an RNA intermediate (class I TEs, retrotransposons) or by a cut-and-paste mechanism (class II TEs, transposons) ${ }^{14}$. Each class is subdivided into many 'superfamilies' based on shared structures, sequence homology, and transposition mechanisms ${ }^{15}$. Class

${ }^{1}$ Key Laboratory of Economic Plants and Biotechnology, Yunnan Key Laboratory for Wild Plant Resources, Kunming Institute of Botany, Chinese Academy of Sciences, Kunming 650201, China. ${ }^{2}$ Institute of Plant Stress Biology, State Key Laboratory of Cotton Biology, Department of Biology, Henan University, Kaifeng 475004, China. ${ }^{3}$ University of the Chinese Academy of Sciences, Beijing 100039, China. ${ }^{4}$ Systematic Botany and Mycology, University of Munich (LMU), Munich 80638, Germany. ${ }^{5}$ State Key Laboratory of Genetic Resources and Evolution, Kunming Institute of Zoology, Chinese Academy of Sciences, Kunming 650223, China. Correspondence and requests for materials should be addressed to J.W. (email: wujianqiang@mail.kib.ac.cn) or G.S. (email: sung@mail.kib.ac.cn) 
I TEs usually cause genome expansion since they can duplicate repeatedly, and they are considered important sources of genome size differences among species ${ }^{16,17}$. Class II TEs exist in the majority of organisms and are the major form of transposable DNA in prokaryotes ${ }^{18}$. Their transposition is catalyzed by transposases that recognize short terminal inverted repeats (TIRs), excise the DNA transposon, and then ligate it into a new target site. In some cases, TEs may lose their transposition ability and are recruited as functional proteins by a process called TE domestication with or without gain of additional domains ${ }^{18}$.

Due to their mobility and capacity of transposition, transposable elements (TEs) are also involved in HGT. Horizontal transfers of both classes of TEs have been reported in fungi and animals, from which 199 transferred TEs were collected by the HTT-DB database with $1 / 3$ involving class II transposons ${ }^{19}$. By searching 40 photosynthetic plant genomes, El Baidouri et al. ${ }^{20}$ recently detected 32 class I LTR retrotransposon transfer events in 26 species, with the transposons in some cases still functional. Some of these transfer events occurred between distantly related lineages, such as palms (Arecaceae) and grapevine (Vitaceae), tomato (Solanaceae) and bean (Fabaceae), or poplar (Salicaceae) and peach (Rosaceae ${ }^{20}$. In contrast, only one horizontal transfer event of a class II transposon was identified in plants, involving two grass species ${ }^{21}$. Here we show that two novel nuclear-encoded transposon genes of class II were transferred from Brassicaceae into Orobanchaceae and are actively transcribed in both genera of Orobanchaceae, although there is little evidence that their homologs in Brassicaceae are transcribed.

\section{Results}

Two hAT transposon genes horizontally acquired by the common ancestor of Orobanche and Phelipanche from Brassicaceae. We developed a pipeline to perform transcriptome screening for foreign genes in $P$. aegyptiaca ${ }^{12}$. Using this screening pipeline, we found that two sequences in the $P$. aegyptiaca transcriptomes exhibited high similarities to the A. thaliana hAT-type DNA transposons: (i) one 1121bp sequence (OrAeBC4_1751) showed 83\% and 75\% identities with AT3G17260 at the amino acid (AA) and nucleotide (NT) level, respectively, while being highly divergent from the most similar sequence in the Phrymaceae Mimulus guttatus (the highest identity was $28 \%$ at the AA level and there was no significant similarity at the NT level); (ii) another 1656-bp fragment (OrAeBC4_65399) had 85\% and 65\% identities with AT3G17290 at AA and NT level, while showing 40\% identity with the most similar homolog in M. guttatus at the AA level and no significant similarity at the NT level (Fig. 1). We then searched the partial genome sequences of Orobanche austrohispanica, O. densiflora, O. gracilis, and O. rapum-genistae $e^{22,23}$ and found that $O$. austrohispanica and O. gracilis also had these two genes; therefore, other species in Orobanche and Phelipanche probably have them as well.

Genomic PCR amplification in seven Orobanche and three Phelipanche species (including P. aegyptiaca) using primers from the conserved regions resulted in amplification of both genes in these 10 species. We then obtained all sequences of Orobanchaceae and submitted to GenBank (Accession numbers: KM037755-6, KT892680-696, KU187277), and multiple sequence alignments showed that the two genes belong to two distinct types and that some gene copies have short indels, indicating that they have evolved into pseudogenes due to frameshift mutations in their coding regions (Fig. 2; Supplementary Fig. S1). All these data indicate that gene transfer events occurred between the common ancestor of Orobanche/Phelipanche and Brassicaceae. The genes were hereafter named $B O$ (Brassicaceae and Orobanchaceae) to reflect their patchy distribution.

To gain insight into the direction of the horizontal transfer and its evolutionary time, we investigated the distribution of the $B O$ transposase genes in the genomes of 18 sequenced species (Supplementary Table S1) that span the core Brassicaceae ${ }^{24,25}$ (320 genera, 3660 species, 49 tribes) as well as the available genomes of other parasitic flowering plants. In most Brassicaceae genome sequences, the $B O$ genes have not been well annotated. We therefore extended the core regions of the $B O$ gene candidates in Brassicaceae species to $5 \mathrm{~kb}$ in both directions and annotated the $B O$ genes manually, using different gene prediction software. ORF Finder and GENSCAN successfully predicted the coding regions of most of the genes. To detect pseudogenized $B O$ genes, we used three additional programs, Augustus, GeneSeqer, and Transeq in the EMBOSS package, and combined multiple sequence alignment with other well-annotated genes for optimal prediction. Except for three species, all analyzed Brassicaceae possess 2 to $11 \mathrm{BO}$ genes (Supplementary Table S1), with half of them apparently pseudogenized, as determined by premature stop codons or indels causing frame shifts (Fig. 2). That we failed to detect the BO genes in three of the species may be due to the incomplete genome sequence data. The same procedure was used to search the genomes of non-Brassicaceae species in the Phytozome database, but no $B O$ genes were found.

To detect $B O$ genes in other parasitic plants, we used the $B O$ genes from $A$. thaliana and $P$. aegyptiaca as queries to BLAST-search relevant transcriptome assemblies, including the assemblies of the Convolvulaceae Cuscuta australis and C. pentagona, the assemblies of the Orobanchaceae Striga hermonthica (StHeBC2), Triphysaria versicolor (TrPuRnBC1) and Triphysaria pusilla (TrVeBC2) in PPGP, the Lauraceae Cassytha filiformis, and the Apodanthaceae Pilostyles thurberi in the $1 \mathrm{KP}$ project (http://onekp.com/project.html). This yielded only sequences with low identities, and subsequent phylogenetic analysis indicated that none of them clustered with the $B O$ genes.

A phylogeny of the protein sequences encoded by the $B O$ transposase genes and proteins from other angiosperms whose genomes have been sequenced is shown in Supplementary Fig. S2. The BO proteins and their relatives form two clusters. Cluster I includes the BOs from Brassicaceae and Orobanchaceae and several sequences from peach (Prunus, Rosaceae). Cluster II contains the remaining angiosperms, the A. thaliana DAYSLEEPER gene $^{26,27}$, and other DAYSLEEPER-like genes in three statistically well-supported groups (Supplementary Fig. S2). Within Cluster I, there are two groups, each containing at least one gene from the Brassicaceae and Orobanchaceae species. A highest posterior probability tree and a maximum likelihood tree obtained from all $B O$ genes of 11 Orobanchaceae and 14 Brassicaceae (the BO genes in Thellungiella parvula were excluded because of their $100 \%$ identity with the homologs from T. halophila) both show distinct BO1 and BO2 clades (Fig. 2). The 
Aratha1 Arabidopsis thaliana Pheaeg1 Phelipanche aegyptiaca Aratha2 Arabidopsis thaliana Pheaeg2 Phelipanche aegyptiaca

Aratha1 Arabidopsis thaliana Pheaeg1 Phelipanche aegyptiaca Aratha2 Arabidopsis thaliana Pheaeg2 Phelipanche aegyptiaca

Aratha1 Arabidopsis thaliana Pheaeg1 Phelipanche aegyptiaca Aratha2 Arabidopsis thaliana Pheaeg2 Phelipanche aegyptiaca

Aratha1 Arabidopsis thaliana Pheaeg1 Phelipanche aegyptiaca Aratha2 Arabidopsis thaliana Pheaeg2 Phelipanche aegyptiaca

Aratha1 Arabidopsis thaliana Pheaeg1 Phelipanche aegyptiaca Aratha2 Arabidopsis thaliana Pheaeg2 Phelipanche aegyptiaca

Aratha1 Arabidopsis thaliana Pheaeg1 Phelipanche aegyptiaca Aratha2 Arabidopsis thaliana Pheaeg2 Phelipanche aegyptiaca

Aratha1 Arabidopsis thaliana Pheaeg1 Phelipanche aegyptiaca Aratha2 Arabidopsis thaliana Pheaeg2 Phelipanche aegyptiaca

Aratha1 Arabidopsis thaliana Pheaeg1 Phelipanche aegyptiaca Aratha2 Arabidopsis thaliana Pheaeg2 Phelipanche aegyptiaca
MRKKLKLDDSLVVD- . - . - . - EEKEAHNAMAKUFI AADVNPKTI NTRSFAKFMSFLGDPKYPPSVSK . . . . . . . . . . . . . . - . . . . - MVI LDMKKKOKLDDS - . - VDD- - EAQEEEKRRYR- SMAKVAI GYGI NPSDENSV- I PEMI HTVC- PDFSLDTSK MEDGDLASGSDVKEKQKSDNEQI VVDSRI PTQAEKEQKYKRSI AGULI RTARVPPHS- . - . . - FMQRMG- PLLPFAVSQ

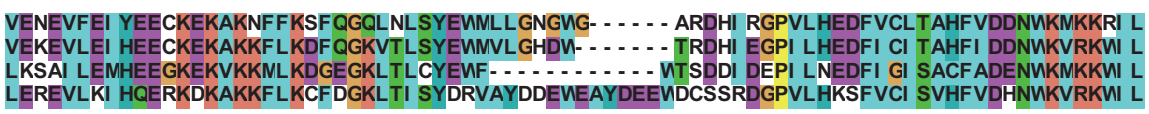

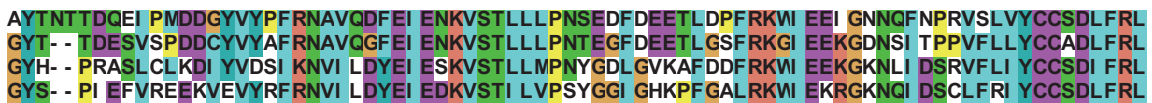

MVDDVLSELSSSLLEDVRMLVGUGRCSATTUNVSLFHLQQAVVLKSEDAFSKDEI YDDYDKPSDEDWI KI QTFCKLTDCI MVDDVFSDLSGSLLEDLRMLVGUGRCSSNNUNVULSNLGRAVDMKNEDEFSKDGI YDDYDKPTDEEWI KI QTYCKLVGCI MVDDMFKDI NI ULMGRVRLLVGUGRMVPTNUDVTLYNLQKAVDMEATNVFEEEEDYQDYEQPSDEEWI KI KTFCKLAGCI MAGDVFDNMDLLLED- I RVLVGUGKMSSTNUNVTLCSLQEALDMEDKKVFAEDAYYQEQDQPSDEDVI MI RTFCKLAGCI
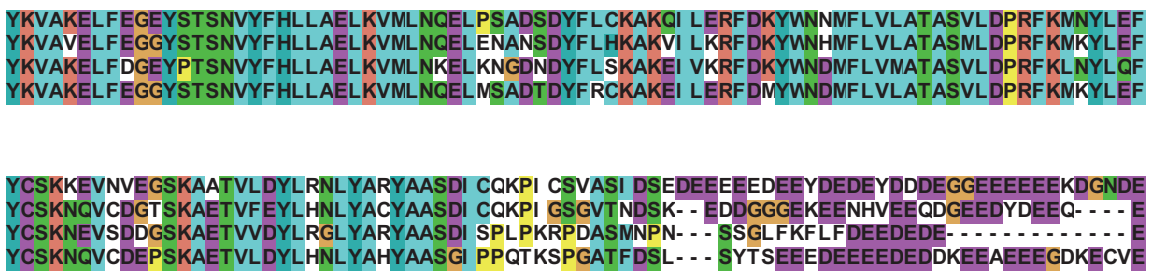

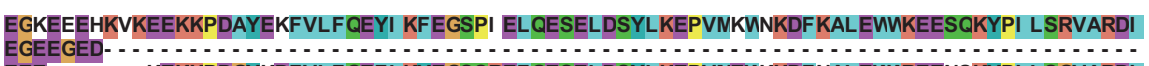
EGEEGED- -1. KEKKPDGYKDFVLFGEFLKYEGSSREFGESELDSYLKEPVMEUKKDFNALEUUREENSKYPI LSGVARDI GGEDKDKEEKKPDAYEDFVLFQEYLKFEESSREFDESEVDSYLKEPVLEMDKDFKALEUUREESQKYPI LSRI ARDI

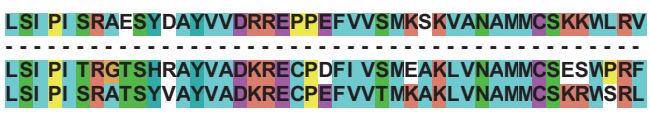

Figure 1. The protein alignment of the two foreign genes from P. aegyptiaca and the most similar proteins from A. thaliana. The species names of Orobanche and Phelipanche are indicated after the sequence IDs. Dashes indicate the sequences were incomplete or gaps introduced in the alignment. Background colors indicate the degree of conservation of the sites.

$B O 1$ genes in Brassicaceae have more copies than do the $\mathrm{BO} 2$ genes, and the $\mathrm{BO} 2$ genes further form two clusters, $B O 2 a$ and $B O 2 b$, each containing genes from most Brassicaceae species, suggesting ancient divergence. Notably, each $B O$ gene clustered with homologs from Sisymbrium irio (Fig. 2). This suggests that the two $B O$ genes may have been laterally transferred into the common ancestor of the Orobanche/Phelipanche clade from Sisymbrium, a small Old World genus that is the sole member of the tribe Sisymbrieae ${ }^{24}$, or from a closely related species.

The $B O$ transposase genes constitute a new member of the $h A T$ transposon superfamily. To gain further insight into the function and evolution of the $B O$ transposase genes, we searched for their domains using InterProScan. Three domains were found: a $h A T$ dimerization, a $h A T$-like transposase, and a ribonuclease $\mathrm{H}$-like domain. The $h A T$ dimerization domain is $\sim 80 \mathrm{AA}$ long and located in the C-terminus; the $h A T$-like transposase domain has $\sim 100 \mathrm{AA}$ and is located in the medial region; the ribonuclease $\mathrm{H}$-like domain is $\sim 500 \mathrm{AA}$ long and covers the above two domains (Fig. 3; Supplementary Fig. S3). The conserved $h A T$ dimerization domain is a hallmark of the $h A T$ transposons and has been shown to be involved in dimerization as well as additional interaction functions ${ }^{28}$. The ribonuclease $\mathrm{H}$-like domain is constituted of a three-layer alpha/beta/alpha fold and exists in some transposases, ribonucleases, exonucleases, and retroviral integrases ${ }^{29}$. Notably, the ribonuclease $\mathrm{H}$-like domain in the BOs contains an insertion of 12-71 AA that lies between the $h A T$-like transposase domain and the hAT dimerization domain (Fig. 3; Supplementary Fig. S3). These results indicate that the BOs belong to a new class of the $h A T$ superfamily.

Given that the BOs are closely related to the A. thaliana DAYSLEEPER protein (Supplementary Fig. S2), which contains an additional $\mathrm{C} 2 \mathrm{H} 2$ type $\mathrm{BED}$-zinc finger domain in the $\mathrm{N}$ terminus ahead of the three classic domains, we searched for this domain in other sequences of cluster II and found that most proteins possess this additional domain. Knip et al. ${ }^{30}$ also identified these genes as DAYSLEEPER-like or SLEEPER. The hAT family members have been categorized as $A c, B u s t e r$, and BuT2, and the DAYSLEEPER protein together with the Ac and TAM3 belongs to the Ac group. To investigate how the BOs are related to these Ac group members, we first used the two BOs in 


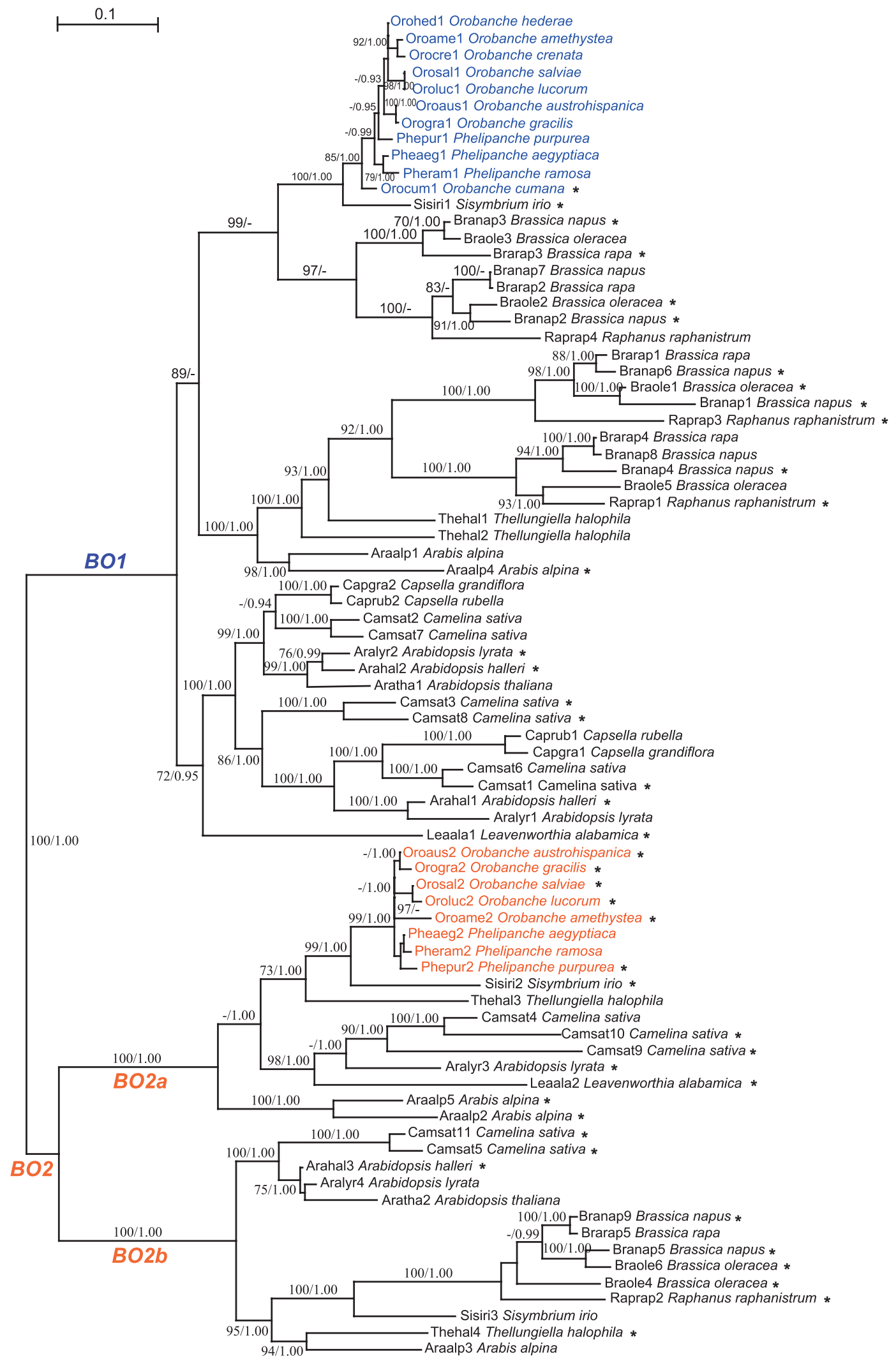

Figure 2. The protein tree of all the BOs in Brassicaceae and Orobanchaceae. Numbers at branches show bootstrap support values for maximum likelihood analysis (before the slash) and posterior probability values for MrBayes analysis (after the slash). Dashes indicate values lower than $70 \%$ in the maximum likelihood analysis and 0.90 in the MrBayes analysis. Asterisks after the species names indicate putative pseudogenes. 
Figure 3. Domain structure comparison of a classic $h A T$ family transposon with the $B O$ transposase gene family in Brassicaceae, Orobanche, and Phelipanche. The ribonuclease H-like domain, the $h A T$-like transposase domain and the $h A T$ dimerization domain are in purple, orange and red, respectively. The insertion position in the ribonuclease $\mathrm{H}$-like domain of the $\mathrm{BO}$ is displayed as a rectangle.
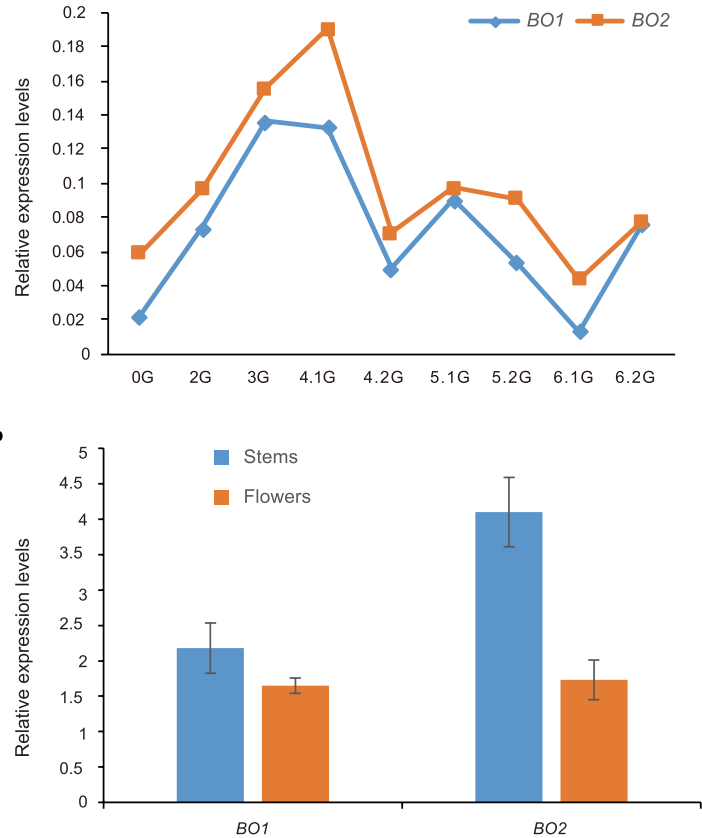

Figure 4. Expression levels of the two BO transposase genes in P. aegyptiaca. (a) Expression levels at different developmental stages (PPGP) estimated from RNA-seq data with ACTIN as the reference gene. (b) Expression levels $( \pm$ SE, $n=3)$ of the two $B O$ genes in stems and flowers assessed by qRT-PCR.

A. thaliana as representatives to compare with the typical Ac of maize and TAM3 of snapdragon. They showed $33 \%$ and $37 \%$ sequence identities with Ac of maize (GenBank accession number ACG45782) and 32\% and 20\% sequence identities with TAM3 of snapdragon (GenBank accession number CAA38906) at AA level. Sequence alignment also showed that the BOs are less similar to Ac and TAM3 than to DAYSLEEPER-like proteins. This supports that the BOs in Orobanche, Phelipanche, and Brassicaceae are a new $h A T$ superfamily member, closely related to the DAYSLEEPER-like genes.

$B O$ genes in $P$. aegyptiaca and $O$. cumana are actively transcribed. The presence of the two $B O$ genes in the RNA-seq data of $P$. aegyptiaca indicates that they are actively transcribed. To determine how the expression level changes in different tissues at different developmental stages, we mapped all clean paired-end reads to our own Trinity assembly of the P. aegyptiaca transcriptome. The FPKM values of the BO1 gene ranged from 2.1 to17.4 and those of the $B O 2$ genes were from 6.0 to 26.5. Figure 4a shows the relative expression levels of $\mathrm{BO} 1$ and $\mathrm{BO} 2$ with actin as the reference gene. Except in flowers, the $\mathrm{BO} 2$ gene had higher expression levels than the $B O 1$ gene in all tissues ${ }^{31}(0 \mathrm{G}$, seeds imbibed, pre-germination; $2 \mathrm{G}$, seedling after exposure to haustorial induction factors; $3 \mathrm{G}$, haustoria attached to host root, early penetration stages, pre-vascular connection; $4.1 \mathrm{G}$, early established parasite, parasite vegetative growth after vascular connection; $4.2 \mathrm{G}$, spider stage; $5.1 \mathrm{G}$, pre-emerged leaves and stems; $5.2 \mathrm{G}$, pre-emerged roots; $6.1 \mathrm{G}$, post emergence from soil - vegetative structures, leaves/stems; $6.2 \mathrm{G}$, post emergence from soil - reproductive structures, floral buds), and a comparison among different development stages showed that both $\mathrm{BO} 1$ and $\mathrm{BO} 2$ exhibited greater expression levels in the $3 \mathrm{G}$ and $4.1 \mathrm{G}$ stages than in the other stages.

We further assessed the transcription levels of the $B O$ transposase genes in flowers and stems of $P$. aegyptiaca by quantitative real time-PCR (qRT-PCR), which provides accurate assessments of gene expression. In flowers 


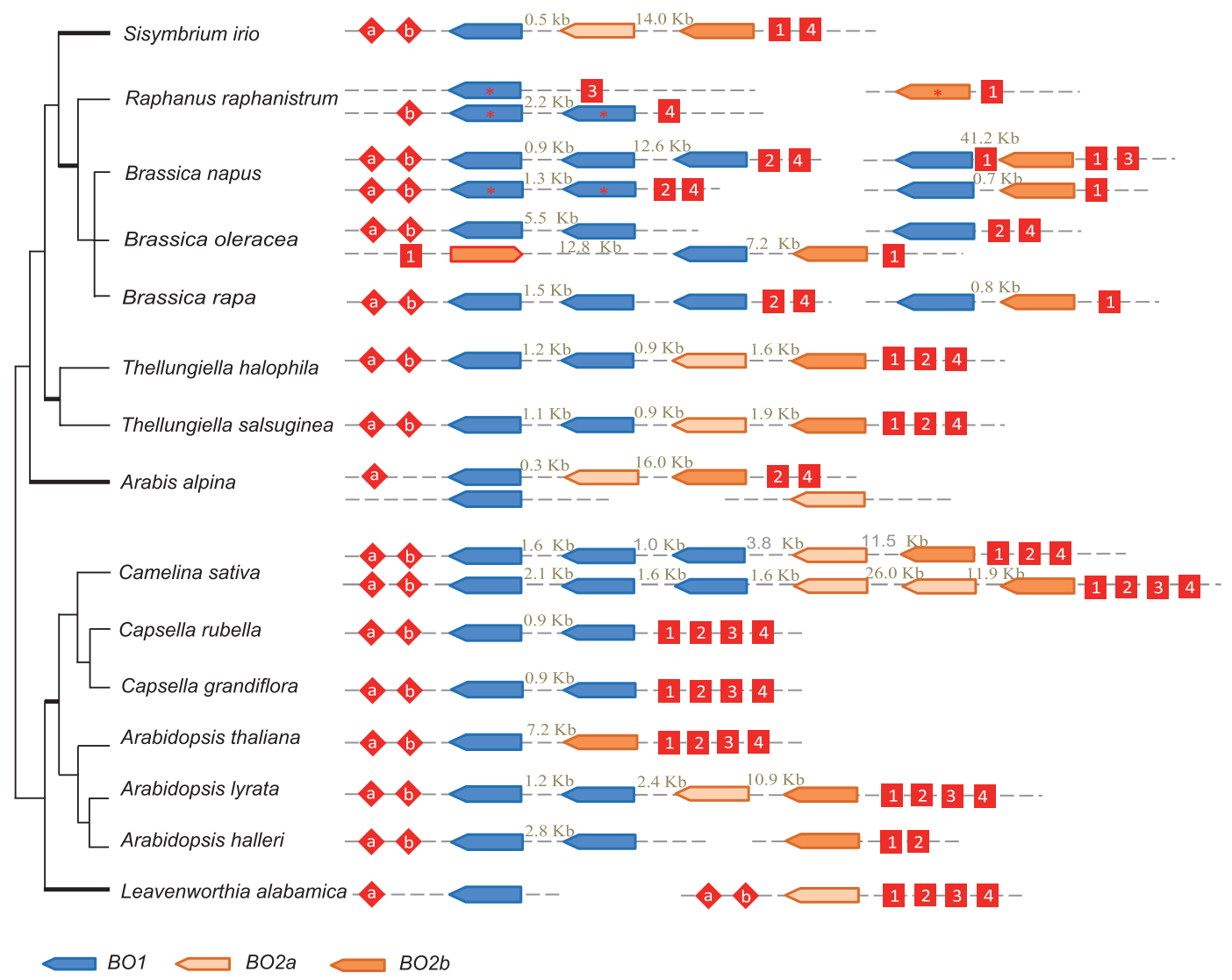

Figure 5. The chromosomal location and synteny of Brassicaceae genes encoding the BO family of $h A T$ transposons. Intergenic regions are indicated above the dash lines. Red rhombuses on the upstream and red rectangles on the downstream flanking regions of the $B O$ genes with letters or numbers indicate the following conserved genes: a, AT3G17205; b, AT3G17240; 1, AT3G17300; 2, AT3G17310; 3, AT3G17320; 4, AT3G17340.

and stems, the expression levels of the two $B O$ genes were similar to those of the reference gene actin $\left(\mathrm{Ct}_{\text {actin }}\right.$ was $24.2 \pm 0.09$ and $25.2 \pm 0.32, \mathrm{Ct}_{B O 1}$ was $23.6 \pm 0.19$ and $24.3 \pm 0.04$, and $\mathrm{Ct}_{B O 2}$ was $25.0 \pm 0.37$ and $24.5 \pm 0.28$ in flowers and stems, respectively). The $B O 1$ gene presented similar expression levels in stems and flowers, while the expression level of the $\mathrm{BO} 2$ gene in stems was more than 1-fold higher than in flowers (Fig. $4 \mathrm{~b}$ ). To determine whether the $B O$ transposase genes are expressed in O. cumana, we performed RT-PCR using the stem and flower tissues with the same primer pair as used in qRT-PCR. It produced a band with the expected size, and the identity of which was confirmed by sequencing.

These transcriptional data indicate that both $B O$ genes have relatively high transcript levels in Phelipanche and Orobanche and that different pathways regulate their transcription.

The $B O$ genes in Brassicaceae show low transcriptional activity. The transcriptional levels of the $B O$ genes in Brassicaceae were investigated by searching the microarray dataset of $A$. thaliana in Genevestigator and the NCBI EST and SRA databases. The Genevestigator analysis indicated that the BOs in A. thaliana are transcribed at low levels in most tissues except that the BO1 gene (AT3G17260) is expressed at medium level in sperm cells (Supplementary Fig. S4). No ESTs corresponding to the two BO genes of A. thaliana were found in the Phytozome database. We further searched multiple RNA-seq datasets of $A$. thaliana (ecotype Col-0) in the SRA database but found no transcription evidence of the two $B O$ genes, except a $B O 2$ RPKM value of 0.48 in one dataset ${ }^{32}$. Data-mining in the Phytozome revealed that two BO1 genes in Capsella rubella (Caprub1 and Caprub2), one BO1 gene (Thehal1) and two BO2 genes in Thellungiella halophila (Thehal3 and Thehal4), and two BO1 genes in Brassica rapa (Brarap1 and Brarap2) have very low expression levels. No expression data were available for $S$. irio, and searching the SRA database indicated that except for two BO1 genes in Brassica napus (Branap6 and Branap7) and all four $B O$ genes in Raphanus raphanistrum (all these genes' RPKM values were $<0.5$ ), other genes had no mapped reads in Brassicaceae. The low transcriptional status, together with pseudogenization in half of the $B O$ genes, suggest that the $B O$ genes in Brassicaceae are generally not actively transcribed and unlikely to be able to transpose.

The $B O$ transposase genes and their flanking regions show synteny in Brassicaceae species. We also investigated the chromosomal location of the $B O$ transposase genes and the synteny of their flanking regions in the 15 available Brassicaceae genomes. Most $B O$ genes were arranged in tandem or very close to each other (Fig. 5). Detailed inspection indicated that the ORF directions are the same for all but one $B O 2$ gene in B. oleracea (Fig. 5). As for the synteny of genes flanking the $B O$ genes, we used six conserved genes with transcript 
activity in the flanking regions of $\mathrm{BO} 1-\mathrm{BO} 2$ in $A$. thaliana to search against the other 14 Brassicaceae genomes. Of the six genes, two are located in the upstream flanking region of the $\mathrm{BO} 1-\mathrm{BO} 2$ structure; the other four genes are located in the downstream flanking region of the $B O 1-B O 2$ structure. This indicates that most flanking genes retained synteny around the $B O$ genes except for a few losses (Fig. 5), such as the two-gene loss in the downstream flanking region of the $B O$ gene in $S$. irio.

To further investigate whether the $B O$ genes in Orobanchaceae and Brassicaceae can transpose, we inspected their flanking regions. Active $h A T$ genes have short flanking regions that are converted to the donor sequence upon insertion (target site duplication, TSD) and short terminal inverted repeats (TIRs) (TSD-TIR-transposase-TIR-TSD). The length of the TSD structure is reported to be $8 \mathrm{bp}$ for the $h A T$ superfamily genes ${ }^{33}$. We failed to obtain the flanking regions of the $B O$ genes in Orobanchaceae and therefore focused on the Brassicaceae $B O$ genes. Transpolator was used to identify the 8-bp TSD and TIR structures in the flanking regions of the Brassicaceae $B O$ genes. Different TSD-TIR structures were found but few had $8 \mathrm{bp}$-long TSDs, nor could their consensus sequence be obtained, including the "(T/C)A(A/G)NG" consensus sequence proposed by Rubin et al $^{33}$. The collinearity of the flanking genes and the absence of the typical TSD-TIR structures suggest that the $B O$ genes in Brassicaceae did not have a history of transposition.

\section{Discussion}

The hAT superfamily (named after the families: hobo from Drosophila melanogaster, Activator (Ac) from maize, and Tam 3 from snapdragon) of class II TEs is widely distributed in fungi, animals, and plants ${ }^{34}$, and phylogenetic analyses suggest that it is ancient, predating the early stages of the divergence of these kingdoms ${ }^{33}$. In animals, $h A T$ has been horizontally transferred among species of Drosophila ${ }^{35-37}$, and a novel $h A T$ transposon having the 8-bp TSDs with the characteristic of recent transposition has been found in the silkworm, Bombyx mori, and in a Triatominae vector of the Chagas parasite, Rhodnius prolixus ${ }^{38}$. Prior to this study, no transfer events involving the transposase genes of the $h A T$ superfamily had been reported in plants. By HGT screening in P. aegyptiaca transcriptomic data, we discovered two $B O$ genes with high similarities to their homologs in Brassicaceae. Because parasitic plants can take up mRNAs from their hosts ${ }^{39}$, foreign mRNAs in P. aegyptiaca might be a contamination from Brassicaceae hosts, since P. aegyptiaca sometimes parasitizes Arabidopsis. To exclude this possibility, we carried out BLAST searches against the draft genomes of four Orobanche species and did genomic PCRs in seven Orobanche and three Phelipanche species, and this revealed two $B O$ genes in the 11 species. Subsequent multiple sequence alignments clearly showed that the $B O$ genes form two clusters, with each present in all tested Orobanche and Phelipanche (Supplementary Fig. S1).

By gene annotation and homology search, we found that the $B O$ genes are absent from bacteria, viruses, and archaea, and are unique to Brassicaceae and the Orobanchaceae genera Orobanche and Phelipanche. Based on phylogenetic analysis of species representing the core Brassicaceae ${ }^{25,40}$, it appears that the two $B O$ genes in Orobanche and Phelipanche are likely to be derived from Sisymbrium, a small Old World genus that is the sole member of Sisymbrieae $e^{24,25}$, or certain closely related species. Future genome sequences of species from the tribes Thelypodieae (26 genera, 224 species) and Isatideae (5 genera, 90 species), which are the closest relatives of Sisymbrieae, will further clarify the origin of the $B O$ genes in Orobanchaceae. It is unlikely that the $B O$ genes were instead transferred from Orobanchaceae to Brassicaceae, given that the $B O$ transcripts were not found in Striga hermonthica (Orobanchaceae) and that the BOs spread from Sisymbrieae (or a close relative) to all the other core Brassicaceae species is implausible.

A scenario explaining the two foreign $B O$ genes in Orobanche and Phelipanche is given in Fig. 6. It assumes a single gene transfer event involving the BO1-BO2 DNA fragment from an ancestor, such as $S$. irio, to the common ancestor of minimally Orobanche and Phelipanche (our sampling of eight Orobanche and three Phelipanche is insufficient to exclude an earlier uptake). Orobanchaceae are a mostly temperate zone family of ca. 2000 species in 89 to 99 genera, and the earliest lineages of Orobanchaceae are not parasitic ${ }^{41}$. Attempts to confirm whether the $\mathrm{BO} 1$ and $\mathrm{BO} 2$ genes in Orobanchaceae are arranged in tandem failed, suggesting either long intervals between the $\mathrm{BO} 1$ and $\mathrm{BO} 2$ genes or that they are located on different chromosomes. Given the possibility of genome structure rearrangement in the recipients, different chromosomal locations or a distant placement on the same chromosome do not rule out an initial simultaneous acquisition.

The gene arrangement $B O 1-B O 2 a-B O 2 b$ likely was present in the ancestor of core Brassicaceae, judging from the analyzed genomes, which represent eight of their 49 tribes of the family and span the root of core Brassicaceae ${ }^{25,40}$. The $B O 2 b$ gene was lost in the Cardamineae; gene duplication and divergence of $B O 1$ occurred in the Camelineae; and gene loss in A. thaliana, A. halleri, and Capsella, with further gene duplication in C. sativa; lineage-specific gene duplication of the $\mathrm{BO} 1$ gene also occurred before the split of Raphanus and Brassica.

The HGT event of $B O$ genes from Brassicaceae to Orobanche and Phelipanche must have occurred before the divergence of Orobanche and Phelipanche. Based on molecular-clock dating, Orobanche and Phelipanche diverged from each other 38 million years ago $(\mathrm{Ma})$, with a $95 \%$ confidence interval of $16-45 \mathrm{Ma}^{23}$. In our $B O$ gene phylogeny, Orobanchaceae genes cluster with Sisymbrium with a stem age of 18 to 25 million years ${ }^{40}$ (Fig. 2; Supplementary Fig. S2). The related tribes Thelypodieae and Isatideae, from whose species genome information is not yet available and who could also have been the donor of the $B O$ genes, shared a common ancestor with Sisymbrieae around 32 to $33 \mathrm{Ma}^{40}$. The HGT might thus have happened between 16 and $33 \mathrm{Ma}$.

Parasite/host plant systems, which all involve distantly related species, are prone to HGT, because of the tight physical connections in the haustoria, which in the case of endoparasites, such as Rafflesia and Pilostyles, involve a network of parenchyma cells living inside the host ${ }^{42,43}$. DNA fragments are therefore transported between parasites and hosts and occasionally become integrated into parasite genomes (Introduction), with end-joining, homologous recombination, and virus-mediated integration as possible mechanisms ${ }^{1,3,4}$. In line with this, the horizontal transfer of TEs has been documented ${ }^{20,21}$, but so far TE-mediated integration of laterally transferred genes has not be found. Such transfer probably is facilitated by the transposons' ability to incorporate into a 


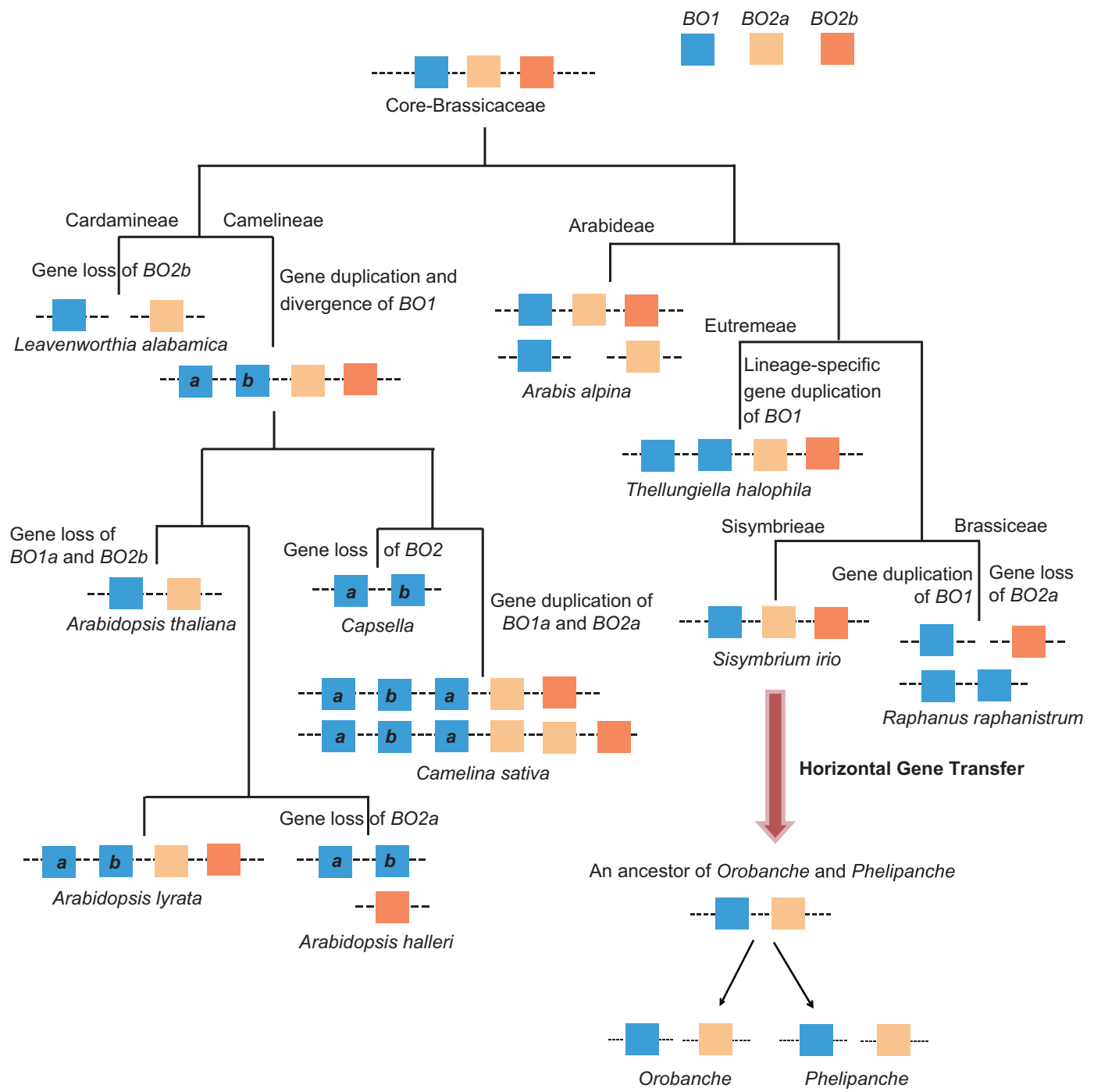

Figure 6. Scenario for the evolutionary history of the $B O$ transposase genes in Brassicaceae and their transfer from an S. irio-like plant to Orobanche and Phelipanche. The genes $B O 1, B O 2 a$ and $B O 2 b$ are indicated as blocks in different colors. The $a$ and $b$ in tribe Camelineae indicate the gene duplication and subsequent divergence of the $\mathrm{BO} 1$ gene. The lineage specific gene duplication of the $\mathrm{BO} 1$ gene in the tribe Eutremeae and the tribes Brassiceae and Sisymbrieae is shown in the same color. The two small unsampled tribes closest to Sisymbrieae are discussed in the text.

recipient genome. This could have involved translation in the recipient cells of transferred $B O$ genes contained in a DNA fragment. In plants, DNA transferred to stem tissue can be passed to the next generation because floral meristems can develop in leaf axils.

The $B O$ genes in Brassicaceae likely lost their transposition ability just after their origin as evidenced by: 1) their flanking regions still being syntenic in almost all the investigated Brassicaceae species, indicating that they did not transpose at all; 2) their lack of the classic TSD border structure required for the recognition of transposases; 3 ) their closest relatives being the SLEEPER genes (Fig. S2), which are domesticated $h A T$ transposons that are involved in the normal plant growth of both $A$. thalian $a^{26,27}$ and rice $\left.{ }^{30} ; 4\right)$ both $B O$ genes in $A$. thaliana being considered domesticated ${ }^{44}$; and lastly by 5 ) many brassicaceous $B O$ genes being pseudogenes (Fig. 2) and all $B O$ s having very little transcriptional activity. Thus, the transfer of these $B O$ genes from Brassicaceae to Orobanchaceae mostly likely did not involve any TE transposition, but instead another mechanism. The relatively high transcript levels of the $B O$ genes in Orobanchaceae could either result from a location in the Orobanchaceae genomes, where transcription is especially active, or from them having gained new functions.

\section{Methods}

Data sources. All transcriptome assemblies of species of Phelipanche (the sole available Phelipanche species in the Parasitic Plant Genome Project (PPGP) is P. aegyptiaca, erroneously called Orobanche aegyptiaca), Striga, and Triphysaria, and the pair-end RNA-seq data from P. aegyptiaca were retrieved from the PPGP website (http:// ppgp.huck.psu.edu). The protein database used by AlienG ${ }^{45}$ for BLAST searches was the NCBI non-redundant 
(nr) database (Sep., 2012) and included all predicted proteins from 22 plant genomes available in the Phytozome database version $9.0^{46}$. The nucleotide database used was the NCBI nucleotide (nt) collection with all predicted transcripts of 22 plant genomes from Phytozome. The databases in the $1 \mathrm{KP}$ project (http://www.onekp.com/ project.html), including the transcriptome assemblies from Cuscuta pentagona Englm., Cassytha filiformis L., and Pilostyles thurberi A. Gray), PlantGDB (http://www.plantgdb.org), and SOL Genomics Network (http:// solgenomics.net), were searched online. Our own RNAseq assembly of Cuscuta australis R.Br. ${ }^{12}$ was searched locally. The genomic sources of the 18 Brassicaceae species are provided in Supplementary Table S1.

Transcriptome screening for foreign genes in Phelipanche aegyptiaca. Transcriptome screening for the transferred genes was performed according to the procedure described previously ${ }^{12}$. Briefly, the contamination from the host transcripts was first excluded by only choosing the genes expressed in seedlings not yet attached to the host; second, the coding regions were predicted, and only sequences with a contiguous amino acid length $\geq 100$ AA were kept for Alien ${ }^{45}$ analysis; finally, after filtering out those with highest similarities to the genes in Mimulus guttatus at the nucleotide acid level, the candidates predicted by AlienG with score ratios of the first non-Lamiales hit to the first Lamiales hit $>1.2$ were retained for manual identification of horizontally transferred genes.

Identification of the foreign $h A T$ transposon in Orobanchaceae and the closest homologs in Brassicaceae and other plants. The foreign genes detected in P. aegyptiaca by searching the PPGP data were two partial gene fragments. To obtain longer sequences, we downloaded the paired-end data from PPGP website and reassembled them using the Trinity software ${ }^{47}$ following the standard procedure. We also searched (BLASTn and tBLASTn) the partial draft genomes from other species of Orobanche, O. densiflora Salz. ex Reut. O. austrohispanica M.J.Y.Foley, O. rapum-genistae Thuill., and O. gracilis Sm. ${ }^{23}$. Hits with identity values $>30 \%$ were extracted and distantly divergent sequences in the phylogeny tree were removed from further analysis. The closest homologs in the genomes of 15 Brassicaceae species were originally found by obtaining the sequence segments using the two BO transposase genes of $A$. thaliana (AT3G17260 and AT3G17290) as queries, which were extended in both directions to $5 \mathrm{~kb}$ for analysis in the next step.

Four types of software were used to identify the genes, including ORF Finder (NCBI) ${ }^{48}$, GENSCAN ${ }^{49}$ (http://genes.mit.edu/GENSCAN.html), Augustus ${ }^{50}$ (http://bioinf.uni-greifswald.de/augustus/submission), Transeq (http://www.ebi.ac.uk/Tools/st/emboss_transeq/), and GeneSeqer ${ }^{51}$ (http://www.plantgdb.org/cgi-bin/ GeneSeqer/index.cgi), with Arabidopsis amino acid sequences as references when necessary. The pseudogenes were determined by the premature stop codons or indels causing coding frame shifts. The same procedure was used to identify the closest homologs with the two $A$. thaliana $B O$ transposase genes in other genomes of relevant species.

Phylogenetic analyses of the new hAT transposases. The protein homologs are extracted from representative species genomes in the Phytozome database and our own assembly of $P$. aegyptiaca by BLAST searching using the two $B O$ transposase genes of $A$. thaliana as queries with the score value threshold set to 40 . The sequences were aligned with ClustalX v2.1 ${ }^{52}$, visually inspected, and manually refined. Gaps and ambiguously sites were removed from the alignment. ModelGenerator (v_851) was used to find the best-fitting model of protein substitution ${ }^{53}$. Phylogenetic trees were inferred under maximum likelihood optimization using PHYML v3. $0^{54}$ and under Bayesian optimization using MrBayes v3.2.5 $5^{55}$. Markov chain Monte Carlo chains were run with the default four chains and using two million generations, sampling trees every 200 generations. After discarding the first 5,000 trees (that is, half the trees) as burn-in, a consensus tree with the posterior probability support values for all clades was calculated from the remaining trees. Trees were viewed and edited using NJplot ${ }^{56}$.

Expression level estimation of $h A T$-like genes in Phelipanche aegyptiaca, Arabidopsis thaliana, and other Brassicaceae. The expression levels of the two foreign $h A T$ transposons in P. aegyptiaca, BO1 and $B O 2$, in different tissues and at different developmental stages were estimated using RSEM ${ }^{57}$ with actin as the reference gene. We mapped all clean reads to the Trinity assembly, and to obtain normalized expression levels we used the fragments per kilo base of exon per million fragments mapped (FPKM) values of the sequences. The actin gene was found with nine transcript isoforms, of which two contained the primer sequences Actin-F/ Actin-R (below) and the sum of their FPKM values was used as the reference. Web-based expression analysis of the two $B O$ transposase genes in A. thaliana (AT3G17260 and AT3G17290) in different tissues, different development stages, and under various treatments was performed using GENEVESTIGATOR v3 (https://www.genevestigator.com $/ \mathrm{gv} /)^{58}$. Estimation of RNAseq-based transcriptional levels was also performed in other Brassicaceae by blast searches against randomly selected SRA datasets.

Genomic PCR amplification of the two BO transposase genes in seven Orobanche and three Phelipanche species. Seeds of P. aegyptiaca and Orobanche cumana Wallr. were inoculated near the roots of 50 days-old tobacco for establishing parasite infection. Two-month-old P. aegyptiaca and O. cumana were sampled and stored at $-80^{\circ} \mathrm{C}$. Samples of the following Orobanchaceae, O. amethystea Thuill., O. crenata Forssk., O. salvia F.W.Schultz, O. hederae Duby, O. lucorum A.Braun ex F.W.Schultz, O. gracilis Sm., Phelipanche purpurea (Jacq.) Soják, and P. ramosa (L.) Pomel were collected in the Munich Botanical Garden. Total DNA was extracted using a modified cetyltrimethylammonium bromide (CTAB) method. For genomic PCR, all $B O 1$ genes were obtained with OhAT-AF2/OhAT-AR primer pair, while the $\mathrm{BO} 2$ genes were amplified by using multiple primer pairs depending on species (Supplementary Table S2). Two primer pairs B2F/B2R and C3F/C3R, were used to get two overlapping regions of the $\mathrm{BO} 2$ in O. cumana. PCR primer sequences and the GenBank accession numbers of the $B O$ genes in the 10 Orobanchaceae species with the herbarium voucher information are indicated in 
Supplementary Table S2. These primers were designed according to the multiple sequence alignment of the $B O$ genes extracted from the transcriptome and draft genomic data above. Amplification products with expected sizes were sequenced directly or after cloning.

qRT-PCR of the BO genes in Phelipanche aegyptiaca and RT-PCR in Orobanche cumana. Fresh tissues of stems and flowers were collected from P. aegyptiaca. Total RNA from each sample was extracted with RNAiso Plus (TaKaRa) following the manufacturer's instructions. RNA concentrations were quantified, and $500 \mathrm{ng}$ of each RNA sample was reverse-transcribed using oligo_(dT) ${ }_{18}$ and RevertAid ${ }^{\mathrm{TM}} \mathrm{H}$ Minus Reverse Transcriptase (Fermentas) in a total volume of $10 \mu \mathrm{L}$. The obtained cDNA samples were diluted to $25 \mu \mathrm{L}$. Specific primer pairs, P.aeg-bo1F/P. aeg-bo1R (5'-AGAACCAAGTCTGTGATGGAACC-3'/5'-TCATTTGTAACACCCGAGCCTAT-3') for the BO1 gene, and bo2F/bo2R (5'-AGTGCCGATACTGATTACTTCCG-3'/5'-GAGGGTTCGTCACAGACTTGGTT- $3^{\prime}$ ) for the $\mathrm{BO} 2$ gene in $P$. aegyptiaca were designed according to their DNA sequences. Actin was again selected as the reference gene for normalizing $\mathrm{cDNA}$ concentration variations $\mathrm{s}^{59}$ and the primer pair Actin-F/Actin-R (5'-CGTGAGAAGATGACGCAGATT-3'/5'-GAACAGCCTGGATAGCAACATAC-3') was designed accordingly. Quantitative real time-PCR (qRT-PCR) was carried out on an CFX ConnectTM Real-Time System (BIO-RAD) using iTaq ${ }^{\mathrm{TM}}$ Universal SYBR Green Supermix (BIO-RAD) following the manufacturer's instructions. The expression levels were calculated using the comparative CT method. Three biological replicates were run for each gene in stems and flowers. Reverse transcription PCR (RT-PCR) was performed to check the expression of the $B O$ transposase genes in O. cumana with the primer pair Actin-F/Actin-R.

TSD-TIR structure search and synteny analysis of genes flanking the $B O$ transposase genes in Brassicaceae. To detect the TSD-TIR-transposase-TIR-TSD structure, genomic sequences covering 5000 $\mathrm{bp}$ in each direction of the $B O$ genes in Brassicaceae species or covering the intergenic segments between two tandem $B O$ genes were retrieved. Within these regions, TIRs with 8-23 bp flanked by 8 bp TSDs were searched by using an updated version of the program Transpolator ${ }^{33}$, with a single imperfect nucleotide in the first base of TIRs allowed and TIRs composed of only two types of nucleotides not considered so as to avoid simple repeats. For the colinearity analysis, six $A$. thaliana genes flanking the two $B O$ genes with transcriptional activity were selected to search their most similar homologs in other Brassicaceae species.

\section{References}

1. Bock, R. The give-and-take of DNA: horizontal gene transfer in plants. Trends Plant Sci. 15, 11-22 (2010).

2. Renner, S. S. \& Bellot, S. Horizontal gene transfer in eukaryotes: fungi-to-plant and plant-to-plant transfers of organellar DNA In Genomics of Chloroplasts and Mitochondria (eds Bock, R. \& Knoop, V.) 223-235 (Springer, Netherlands, 2012).

3. Gao, C. et al. Horizontal gene transfer in plants. Funct. Integr. Genomics 14, $23-29$ (2014).

4. Keeling, P. J. Functional and ecological impacts of horizontal gene transfer in eukaryotes. Curr. Opin. Genet. Dev. 19, 613-619 (2009).

5. Li, F. W. et al. Horizontal transfer of an adaptive chimeric photoreceptor from bryophytes to ferns. Proc. Natl. Acad. Sci. USA 111, 6672-6677 (2014).

6. Xi, Z. et al. Horizontal transfer of expressed genes in a parasitic flowering plant. BMC Genomics 13, 227 (2012).

7. Rice, D. W. et al. Horizontal transfer of entire genomes via mitochondrial fusion in the angiosperm Amborella. Science 342, $1468-1473$ (2013).

8. Xi, Z. et al. Massive mitochondrial gene transfer in a parasitic flowering plant clade. PLoS Genetics 9, e1003265 (2013).

9. Sun, T. et al. An acyltransferase gene that putatively functions in anthocyanin modification was horizontally transferred from Fabaceae into the genus Cuscuta. Plant Divers, doi: 10.1016/j.pld.2016.04.002 (2016).

10. Yoshida, S., Maruyama, S., Nozaki, H. \& Shirasu, K. Horizontal gene transfer by the parasitic plant Striga hermonthica. Science 328, $1128(2010)$.

11. Zhang, Y. et al. Evolution of a horizontally acquired legume gene, albumin 1, in the parasitic plant Phelipanche aegyptiaca and related species. BMC Evol. Biol. 13, 48 (2013).

12. Zhang, D. L. et al. Root parasitic plant Orobanche aegyptiaca and shoot parasitic plant Cuscuta australis obtained Brassicaceaespecific strictosidine synthase-like genes by horizontal gene transfer. BMC Plant Biol. 14, 19 (2014).

13. Kim, G., LeBlanc, M. L., Wafula, E. K., Depamphilis, C. W. \& Westwood, J. H. Genomic-scale exchange of mRNA between a parasitic plant and its hosts. Science 345, 808-811 (2014).

14. Wicker, T. et al. A unified classification system for eukaryotic transposable elements. Nat. Rev. Genet. 8, 973-982 (2007).

15. Bennetzen, J. L. Transposable element contributions to plant gene and genome evolution. Plant Mol.Biol. 42, 251-269 (2000).

16. Casacuberta, E. \& Gonzalez, J. The impact of transposable elements in environmental adaptation. Mol. Ecol. 22, 1503-1517 (2013).

17. Bennetzen, J. L. \& Wang, H. The contributions of transposable elements to the structure, function, and evolution of plant genomes. Annu. Rev. Plant Biol. 65, 505-530 (2014).

18. Munoz-Lopez, M. \& Garcia-Perez, J. L. DNA transposons: nature and applications in genomics. Curr. Genomics 11, 115-128 (2010).

19. Dotto, B. R. et al. HTT-DB: horizontally transferred transposable elements database. Bioinformatics 31, 2915-2917 (2015).

20. El Baidouri, M. et al. Widespread and frequent horizontal transfers of transposable elements in plants. Genome Res. 24, 831-838 (2014).

21. Diao, X., Freeling, M. \& Lisch, D. Horizontal transfer of a plant transposon. PLoS Biol. 4, 119-128 (2006).

22. Piednoel, M. et al. Next-generation sequencing reveals the impact of repetitive DNA across phylogenetically closely related genomes of Orobanchaceae. Mol. Biol. Evol. 29, 3601-3611 (2012).

23. Piednoel, M., Sousa, A. \& Renner, S. S. Transposable elements in a clade of three tetraploids and a diploid relative, focusing on Gypsy amplification. Mob. DNA 6, 5 (2015).

24. Al-Shehbaz, I. A. A generic and tribal synopsis of the Brassicaceae (Cruciferae). Taxon 61, 931-954 (2012).

25. Koch, M. A. et al. BrassiBase: Tools and biological resources to study characters and traits in the Brassicaceae-version 1.1. Taxon 61, 1001-1009 (2012).

26. Bundock, P. \& Hooykaas, P. An Arabidopsis hAT-like transposase is essential for plant development. Nature 436, $282-284$ (2005).

27. Knip, M. et al. DAYSLEEPER: a nuclear and vesicular-localized protein that is expressed in proliferating tissues. BMC Plant Biol. 13, 211 (2013).

28. Essers, L., Adolphs, R. H. \& Kunze, R. A highly conserved domain of the maize activator transposase is involved in dimerization. Plant Cell 12, 211-223 (2000). 
29. Gough, J., Karplus, K., Hughey, R. \& Chothia, C. Assignment of homology to genome sequences using a library of hidden Markov models that represent all proteins of known structure. J. Mol. Biol. 313, 903-919 (2001).

30. Knip, M., de Pater, S. \& Hooykaas, P. J. The SLEEPER genes: a transposase-derived angiosperm-specific gene family. BMC Plant Biol. 12, 192 (2012).

31. Westwood, J. H. et al. The parasitic plant genome project: new tools for understanding the biology of Orobanche and Striga. Weed Sci. 60, 295-306 (2012).

32. Clauw, P. et al. Leaf responses to mild drought stress in natural variants of Arabidopsis. Plant Physiol. 167, 800-816 (2015).

33. Rubin, E., Lithwick, G. \& Levy, A. A. Structure and evolution of the hAT transposon superfamily. Genetics 158, $949-957$ (2001).

34. Kempken, F. \& Windhofer, F. The $h A T$ family: a versatile transposon group common to plants, fungi, animals, and man. Chromosoma 110, 1-9 (2001).

35. Depra, M., Panzera, Y., Ludwig, A., Valente, V. L. \& Loreto, E. L. Hosimary: a new $h A T$ transposon group involved in horizontal transfer. Mol. Genet. Genomics 283, 451-459 (2010).

36. Schaack, S., Gilbert, C. \& Feschotte, C. Promiscuous DNA: horizontal transfer of transposable elements and why it matters for eukaryotic evolution. Trends. Ecol. Evol. 25, 537-546 (2010).

37. Rossato, D. O. et al. BuT2 is a member of the third major group of $h A T$ transposons and is involved in horizontal transfer events in the genus Drosophila. Genome Biol. Evol. 6, 352-365 (2014).

38. Zhang, H. H. et al. A novel hAT element in Bombyx mori and Rhodnius prolixus: its relationship with miniature inverted repeat transposable elements (MITEs) and horizontal transfer. Insect Mol. Biol. 22, 584-596 (2013).

39. Roney, J. K., Khatibi, P. A. \& Westwood, J. H. Cross-species translocation of mRNA from host plants into the parasitic plant dodder. Plant Physiol. 143, 1037-1043 (2007).

40. Beilstein, M. A., Nagalingum, N. S., Clements, M. D., Manchester, S. R. \& Mathews, S. Dated molecular phylogenies indicate a Miocene origin for Arabidopsis thaliana. Proc. Natl. Acad. Sci. USA 107, 18724-18728 (2010).

41. Schneeweiss, G. M. Phylogenetic relationships and evolutionary trends in Orobanchaceae in Parasitic Orobanchaceae (eds Joel, M. D., Gressel, J. \& Musselman, J. L.) 243-265 (Berlin Heidelberg, 2013).

42. Nikolov, L. A. et al. Holoparasitic Rafflesiaceae possess the most reduced endophytes and yet give rise to the world's largest flowers. Ann. Bot. 114, 233-242 (2014).

43. Bellot, S. \& Renner, S. S. The plastomes of two species in the endoparasite genus Pilostyles (Apodanthaceae) each retain just five or six possibly functional genes. Genome Biol. Evol. 8, 189-201 (2016).

44. Hoen, D. R. \& Bureau, T. E. Discovery of novel genes derived from transposable elements using integrative genomic analysis. Mol. Biol. Evol. 32, 1487-1506 (2015).

45. Tian, J. et al. AlienG: an effective computational tool for phylogenetic identification of horizontally transferred genes. [Zhu Q. (ed.)] [23-25] Proceedings of the The third international conference on bioinformatics and computational biology (BICoB). (New Orleans, Louisiana USA, 2011).

46. Goodstein, D. M. et al. Phytozome: a comparative platform for green plant genomics. Nucleic Acids Res. 40, D1178-D1186 (2012).

47. Grabherr, M. G. et al. Full-length transcriptome assembly from RNA-Seq data without a reference genome. Nat. Biotechnol. 29, 644-652 (2011).

48. Rombel, I. T., Sykes, K. F., Rayner, S. \& Johnston, S. A. ORF-FINDER: a vector for high-throughput gene identification. Gene 282, $33-41$ (2002).

49. Burge, C. \& Karlin, S. Prediction of complete gene structures in human genomic DNA. J. Mol. Biol. 268, 78-94 (1997).

50. Stanke, M. \& Morgenstern, B. AUGUSTUS: a web server for gene prediction in eukaryotes that allows user-defined constraints. Nucleic Acids Res. 33, W465-W467 (2005).

51. Schlueter, S. D. GeneSeqer@PlantGDB: gene structure prediction in plant genomes. Nucleic Acids Res. 31, 3597-3600 (2003).

52. Thompson, J. D., Gibson, T. J., Plewniak, F., Jeanmougin, F. \& Higgins, D. G. The CLUSTAL_X windows interface: flexible strategies for multiple sequence alignment aided by quality analysis tools. Nucleic Acids Res. 25, 4876-4882 (1997).

53. Keane, T. M., Creevey, C. J., Pentony, M. M., Naughton, T. J. \& McLnerney, J. O. Assessment of methods for amino acid matrix selection and their use on empirical data shows that ad hoc assumptions for choice of matrix are not justified. BMC Evol. Biol. 6 (2006).

54. Guindon, S. \& Gascuel, O. A simple, fast, and accurate algorithm to estimate large phylogenies by maximum likelihood. Syst. Biol. 52, 696-704 (2003).

55. Ronquist, F. \& Huelsenbeck, J. P. MrBayes 3: Bayesian phylogenetic inference under mixed models. Bioinformatics 19, 1572-1574 (2003).

56. Perriere, G. \& Gouy, M. WWW-query: an on-line retrieval system for biological sequence banks. Biochimie 78, 364-369 (1996)

57. Li, B. \& Dewey, C. N. RSEM: accurate transcript quantification from RNA-Seq data with or without a reference genome. BMC Bioinformatics 12, 323 (2011)

58. Hruz, T. et al. Genevestigator v3: a reference expression database for the meta-analysis of transcriptomes. Adv. Bioinformatics 2008, 420747 (2008).

59. Gonzalez-Verdejo, C. I. et al. Selection of housekeeping genes for normalization by real-time RT-PCR: analysis of Or-MYB1 gene expression in Orobanche ramosa development. Anal. Biochem. 379, 176-181 (2008).

\section{Acknowledgements}

We thank Prof. Yongqing Ma (College of Resources and Environment, Northwest A\&F University, Yangling, Shaanxi Province, China) for P. aegyptiaca and O. cumana seeds, the PPGP project (Virginia Tech, Penn State University, University of Virginia, and University of California, Davis) for the transcriptome data for Orobanche aegyptiaca (now named Phelipanche aegyptiaca), Triphysaria versicolor, and Striga hermonthica, and the 1 KP project (Gane Ka-Shu Wong, University of Alberta) for the transcriptome data for Cuscuta, Cassytha, and Pilostyles. We thank N. Cusimano (Systematic Botany and Mycology, University of Munich, Germany) for BLAST searching draft genomes of Orobanche, and E. Rubin and A. A. Levy for the Transpolator program. This work was supported by the National Natural Science Foundation of China (No. 31301037 and 31470012 to G.S.), the Natural Science Foundation of Yunnan Province of China (No. 2013FB068 to G.S.), the Young Academic and Technical Leader Raising Foundation of Yunnan Province (No. 2014HB046 to G.S.), the Western Light Talent Culture Project of the Chinese Academy of Sciences (to G.S.), the Strategic Priority Research Program of the Chinese Academy of Sciences (No. XDB1 1050200 to J.W.), and the Yunnan Recruitment Program of Experts in Sciences (2012HA016 to J.W.), and the Open Foundation of the State Key Laboratory of Genetics Resources and Evolution, CAS.

\section{Author Contributions}

G.S. conceived and designed the study. T.S., G.S., Y.X. and Q.Y. generated the data and performed the analysis. S.S.R. contributed materials. T.S., G.S., S.S.R. and J.W. wrote the paper. All authors read and approved the final manuscript. 


\section{Additional Information}

Accession codes: Sequence data for the Orobancheceae PCR amplification genes have been deposited in the GenBank database (http://www.ncbi.nlm.nih.gov/) under accession number KM037755, KM037756, KT892680698 and KU187277.

Supplementary information accompanies this paper at http://www.nature.com/srep

Competing financial interests: The authors declare no competing financial interests.

How to cite this article: Sun, T. et al. Two $h A T$ transposon genes were transferred from Brassicaceae to broomrapes and are actively expressed in some recipients. Sci. Rep. 6, 30192; doi: 10.1038/srep30192 (2016).

(c) (i) This work is licensed under a Creative Commons Attribution 4.0 International License. The images or other third party material in this article are included in the article's Creative Commons license, unless indicated otherwise in the credit line; if the material is not included under the Creative Commons license, users will need to obtain permission from the license holder to reproduce the material. To view a copy of this license, visit http://creativecommons.org/licenses/by/4.0/ 